\title{
Coronal shocks associated with CMEs and flares and their space weather consequences
}

\author{
Marina Laskari ${ }^{1}$, Panagiota Preka-Papadema ${ }^{1}$, Constantine \\ Caroubalos $^{2}$, George Pothitakis ${ }^{2}$, Xenophon Moussas ${ }^{1}$, Eleftheria \\ Mitsakou $^{1}$ and A. Hillaris ${ }^{1}$ \\ ${ }^{1}$ Department of Physics, University of Athens, 15784 Athens, Greece \\ ${ }^{2}$ Department of Informatics, University of Athens, 15783 Athens, Greece
}

\begin{abstract}
We study the geoeffectiveness of a sample of complex events; each includes a coronal type II burst, accompanied by a GOES SXR flare and LASCO CME. The radio bursts were recorded by the ARTEMIS-IV radio spectrograph, in the 100-650 MHz range; the GOES SXR flares and SOHO/LASCO CMEs, were obtained from the Solar Geophysical Data (SGD) and the LASCO catalogue respectively. These are compared with changes of solar wind parameters and geomagnetic indices in order to establish a relationship between solar energetic events and their effects on geomagnetic activity.
\end{abstract}

Keywords. Sun: coronal mass ejections (CMEs), Sun: flares, Sun: radio radiation, (Sun:) solarterrestrial relations

\section{Introduction}

The primary sources of geomagnetic phenomena are the solar eruptive events; they initiate the disturbances of solar wind parameters (magnetic field, speed, density and temperature) which in turn drive the Space Weather effects. The latter is characterized by a geomagnetic index such as the Disturbance storm time or Dst (cf. Gopalswamy et al. 2007; the geomagnetic field variations are, on the other hand, quantified, on average, by the $\mathbf{K p}$ index. Though the connection of the geomagnetic phenomena with processes on the Sun is well established, the geomagnetic storm effectiveness of CMEs and solar flares is still an open question as published results are not conclusive (for a review cf. Yermolaev et al. 2005, also Yermolaev \& Yermolaev 2006).

We study the geoeffectiveness of a medium size sample of solar events; each includes a coronal type II burst sometimes extending to an interplanetary type II, accompanied by a GOES SXR flare and SOHO/LASCO CME. The radio bursts were recorded by the ARTEMIS-IV radio spectrograph (Caroubalos et al. 2001); the GOES SXR flares and SOHO/LASCO CMEs, were obtained from the Solar Geophysical Data (SGD) and the $\mathrm{SOHO} / \mathrm{LASCO}$ lists respectively.

We examine the effects on the solar wind parameters and the $\mathbf{K p}$, Dst geomagnetic indices variations two to three days after the events recording.

\section{Data selection \& analysis}

The ARTEMIS IV radiospectrograph (Caroubalos et al. 2001) observed about 40 type II and/or IV radio bursts which they were published in the form of a catalogue (Caroubalos et al. 2004). From this catalogue we have used in our study the same fourteen events studied by Pothitakis et al. (these proceedings); here we adopted the same numbering of events. The solar wind parameters (magnetic field (B and Bz component), speed (V), 

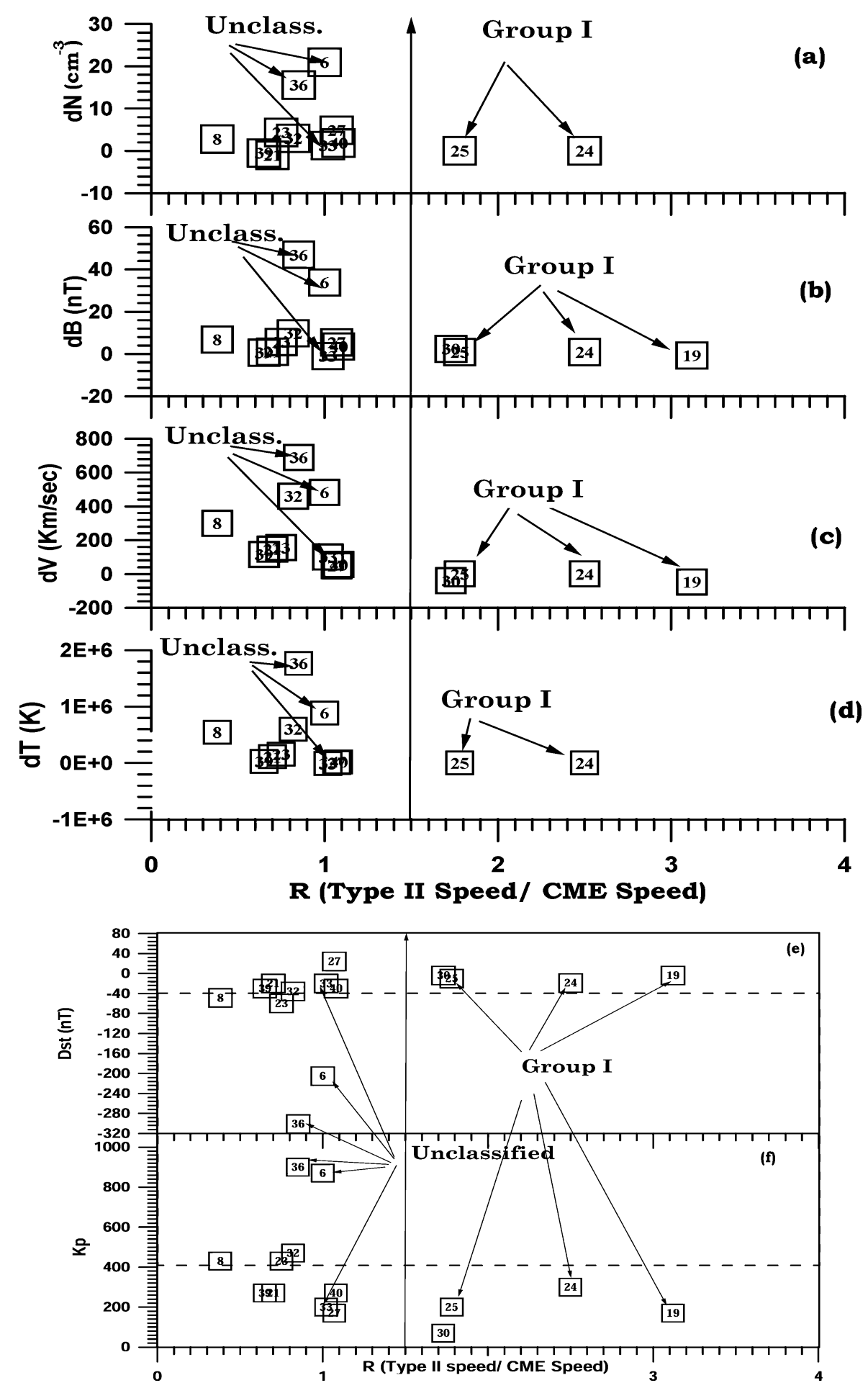

Figure 1. Solar Wind Parameters Variation \& Space Weather indices as a function of the ratio of the speed of Type II to the CME speed $\left(V_{r}=V_{I I} / V_{C M E}\right)$ : (a)Density (dN) in $\mathrm{cm}^{-3}$, (b) Magnetic Field (dB) in nT, (c) Speed (dV) in Km/sec, (d) Temperature (dT) in K, (e) Dst, (f) Kp. (We have labeled Events of Group I \& Unclassified (cf. Table 1 of Pothitakis et al. in these Proceedings); The quiet Solar Wind Parameters at $1 \mathrm{AU}$ were: $\mathrm{B}=5 \mathrm{nT}, \mathrm{V}=350 \mathrm{Km} / \mathrm{sec}$, $\mathrm{N}=5 \mathrm{~cm}^{-3}, \mathrm{~T}=10^{5} \mathrm{~K}$.) 
temperature $(\mathrm{T})$ and density $(\mathrm{N}))$ at 1 A.U., and the geomagnetic indices Kp and Dst were obtained from the OMNI data base.

The data set was carefully selected in order to represent solar events within periods of relative inactivity (deduced from the GOES SXR flux 3-4 days prior and after the event) in order to establish an association between the space weather effects and the solar driver.

In Pothitakis et al. (cf. their Table 1) the association of the flare-CME-Type II burst parameters was studied; in this report on the other hand, we examine the variation of the geomagnetic indices Dst \& Kp and of the Solar Wind Parameters (dB, dV, dT, dN)) with the ratio of the speed of Type II to the CME speed $\left(V_{r}=V_{I I} / V_{C M E}\right)$; this ratio provides a sort of indication of the CME capability in driving a magnetohydrodynamic shock. The results of this comparison are plotted in Figure 1.

Our examination indicates that a certain class of events may initiate space storms (Events 23, 06, 36, 08 \& 32) give $\mathbf{D s t}<-40$ or $\mathbf{K p}>400$ ), the Bastille Event (36) among them.

Comparing this result with the classification of Pothitakis et al. we note that the most geoeffective events of our data sample are from Group II $(23,08,32)$ plus two of the events which were not classified $(36,06)$. The events in Group I $(24,25,19)$, on the other hand, include fast coronal shocks $(1213-1940 \mathrm{~km} / \mathrm{sec})$ and CMEs with speeds in the $400-800 \mathrm{~km} / \mathrm{sec}$ range yet their effect on space weather are small.

Lastly, we note that there is a trend of increased geoeffectiveness and Solar Wind Variations with $V_{r}$ but only when $V_{r}<1.5$.

\section{Final remarks}

We have studied the geoeffectiveness of a medium size sample of complex events, each including a coronal type II burst, a GOES SXR flare and a SOHO/LASCO CME; the results were compared with the classification of Pothitakis et al. (these proceedings) which was based on parameters, related to shock \& CME kinetics and radio bursts-flare-CME timing.

Fast coronal shocks do not, always, initiate storms, yet fast shocks in the front of fast CMEs have a higher probability of inciting disturbances in the near earth environment. Furthermore, a trend of increase in geomagnetic effects with $\mathrm{R}$ was found when $V_{r}<1.5$. This, in fact, excludes fast coronal shocks accompanied by relatively slow CMEs.

\section{References}

Caroubalos, C., Maroulis, D., Patavalis, N., Bougeret, J. L., Dumas, G., Perche, C., Alissandrakis, C., Hillaris, A., Moussas, X., Preka-Papadema, P., Kontogeorgos, A., Tsitsipis, P., \& Kanelakis, G., 2001, Exp. Astron., 11, 23

Caroubalos, C., Hillaris, A., Bouratzis, C., Alissandrakis, C. E., Preka-Papadema, P., Polygiannakis, J., Tsitsipis, P., Kontogeorgos, A., Moussas, X., Bougeret, J. L., Dumas, G., \& Perche, C., 2004, A\&BA, 413, 1125

Gopalswamy, N., Yashiro, S., \& Akiyama, S., 2007, J. Geophys. Res., 112(11), 6112

Yermolaev, Y. I., Yermolaev, M. Y., Zastenker, G. N., Zelenyi, L. M., Petrukovich, A. A., \& Sauvaud, J. A., 2005, Planetary and Space Science, 53, 189

Yermolaev, Y. I. \& Yermolaev, M. Y., 2006, Adv. Sp. Res., 37, 1175 